\title{
Chaire de relations publiques et communication marketing
}

Faculté de communication - Université du Québec à Montréal (UQÀM)

Benoît Cordelier

\section{OpenEdition}

\section{Journals}

Édition électronique

URL : http://journals.openedition.org/communicationorganisation/535

DOI : 10.4000/communicationorganisation.535

ISSN : 1775-3546

Éditeur

Presses universitaires de Bordeaux

Édition imprimée

Date de publication : 1 juin 2008

Pagination : 133-137

ISBN : 978-2-86787-506-5

ISSN : $1168-5549$

\section{Référence électronique}

Benoît Cordelier, "Chaire de relations publiques et communication marketing », Communication et organisation [En ligne], 33 | 2008, mis en ligne le 01 juin 2011, consulté le 21 septembre 2020. URL http://journals.openedition.org/communicationorganisation/535; DOI : https://doi.org/10.4000/ communicationorganisation.535 


\section{Chaire de relations publiques et communication marketing Faculté de communication Université du Québec à Montréal (UQÀM)}

Benoit Cordelier

cordelier.benoit@uqam.ca

\section{Historique}

En 1996, est créé à l'UQÀM le premier programme universitaire complet en relations publiques du Québec. Dès 1999, la nécessité à la fois d'exploiter et d'alimenter les connaissances produites dans cet environnement pousse Danielle Maisonneuve, professeur du département de communication sociale et publique, à travailler à la concrétisation d'une structure de recherche. Celle-ci est finalement fondée en 2002 avec une volonté forte d'ancrage dans la pratique professionnelle. Elle s'appellera Chaire en relations publiques. Cette dernière expression est bien évidemment à comprendre dans la tradition nord-américaine et à rapprocher de la notion de communication des organisations, plus répandue en France.

La recherche y est envisagée comme un moyen de contribuer au débat public sur les questions et les enjeux liés aux conditions, à la pratique et au rôle des relations publiques dans la société. En effet, la Chaire a pour objectifs non seulement de faire de la recherche fondamentale et appliquée, mais également de travailler au partage des connaissances que ce soit par des publications, des conférences et des colloques ou encore par un travail relationnel fort avec le milieu professionnel. Ce travail de diffusion se fait par exemple à travers la publication d'ouvrages dans la collection Communication-Relation publiques dirigée par Danielle Maisonneuve et éditée aux Presses de l'Université du Québec.

2007 voit la création d'une formation bi-facultaire proposée conjointement par la Faculté de communication et l'Ecole des Sciences de la Gestion de l'UQÀM: le baccalauréat en communication marketing, premier cursus du genre au Québec, créé à l'initiative de l'Association des agences de publicité du Québec 


\section{Etat de la recherche}

(AAPQ). A cette occasion et suite également à la création du Centre d'études AAPQ-Infopresse sur les communications marketing, en décembre 2007 la Chaire de relations publiques change de nom pour s'appeler désormais Chaire de relations publiques et communication marketing.

En janvier 2008, Danielle Maisonneuve passe alors le relais à Bernard Motulsky qui est nommé titulaire de la Chaire pour un mandat allant jusqu'en mars 2010.

\section{La structure de recherche}

Centre d'études CNW Telbec sur les relations de presse

Le Centre d'études s'occupe de recherche, d'amélioration de l'enseignement et de diffusion publique des nouvelles connaissances sur les relations avec la presse (par des publications, des présentations, des stages, etc.). Il vise à améliorer l'enseignement sur les voies de communication entre les relationnistes et les représentants des médias, telles la diffusion sur internet et la diffusion push et pull d'information, dans un contexte de mondialisation et de concentration de la presse.

Centre d'études sur les communications municipales

Le Centre réalise des recherches sur les communications civiques de proximité, c'est-à-dire les communications qui émergent du nouveau paysage social reliant les citoyens, les élus et les employés municipaux. Il réalise également des études sur la communication des risques, la communication en situation d'urgence (portée et efficacité des messages municipaux devant être divulgués à l'occasion de la gestion d'une crise ou d'un sinistre). publiques

Centre d'études national sur l'industrie des relations Le Centre d'études désire comprendre, analyser, mesurer, voire anticiper les changements qui s'opèrent au sein de l'industrie canadienne des relations publiques. Les études que mène ce Centre contribuent à approfondir la connaissance des perceptions des dirigeants d'entreprise à l'égard des relations publiques. Par exemple, l'opinion et les attentes des clients des firmes de relations publiques sont étudiées par les chercheurs du Centre.

Centre d'études Journal de Montréal sur les communications d'affaires

Le Centre développe les connaissances reliées aux communications d'affaires au Québec : il collabore avec le cahier «Votre argent » du Journal de Montréal ainsi que la Chambre de Commerce du Montréal Métropolitain pour la réalisation d'études réalisées auprès de la 
communauté des affaires. Le Centre vise à atteindre une meilleure compréhension et une connaissance plus approfondie du point de vue des leaders de la communauté des affaires sur une variété d'enjeux, du point de vue des communications internes aussi bien qu'externes, qu'entretiennent les entreprises avec leurs différents publics.

Centre d'études ODESIA sur les nouvelles technologies et les relations publiques

Le Centre d'études réalise des recherches ayant pour objet l'impact qu'ont les technologies de l'information et des communications (TIC) sur les pratiques reliées aux relations publiques. Il contribue à enrichir la réflexion sur les usages de ces technologies autant par les communicateurs que par les divers publics cibles et parties prenantes qui sont en relation avec les organisations. Il oeuvre également au développement de ressources et d'outils favorisant le succès des interventions de relations publiques dans une perspective dialogique.

\section{Laboratoire d'analyse de presse Caisse, Chartier}

Le Laboratoire contribue à l'amélioration des connaissances dans l'étude du discours de presse en développant une méthode d'analyse propre au Laboratoire et participe à la formation des chercheurs et des étudiants qui s'intéressent à ce domaine du savoir.

Centre d'études sur les responsabilités sociales, le développement durable et l'éthique

Face au mouvement continu de mondialisation des marchés et au besoin de compétitivité des organisations, comment considérer les responsabilités sociales des entreprises vis-à-vis leurs environnements interne et externe, ainsi qu'envers la communauté locale et internationale? Le Centre d'études s'intéresse aux différentes préoccupations entourant les questions de responsabilités sociales, d'éthique et de développement durable dans les organisations.

\section{Laboratoire Trans-com}

Le Laboratoire permet de réaliser des modèles opérationnels à partir d'observations et d'analyse des pratiques communicationnelles. Ces modèles permettent de transférer, en les acteurs d'un même milieu ou entre ceux de différents domaines d'activités les fondements de leurs stratégies professionnelles et les éléments de leurs tactiques opérationnelles.

Centre d'études sur le protocole

Le Centre vise la recherche et la diffusion des connaissances en matière de protocole diplomatique et d'usages sociaux. Il entend en favoriser l'étude sous l'angle de la communication, des relations 


\section{Etat de la recherche}

internationales, de la science politique, de l'histoire, de la géographie culturelle et de la gouvernance. Il se propose également de mettre en valeur les pratiques qui ont cours au sein des institutions publiques et des organisations internationales. marketing

Centre d'études AAPQ-Infopresse sur les communications C'est le dernier centre à avoir été créé. Les axes de recherche s'orientent vers l'éthique en publicité, les normes de qualité dans l'industrie publicitaire, internet et la communication marketing, mondialisation de la communication marketing, l'analyse critique du phénomène publicitaire.

\section{Les projets de recherche en cours} Motulsky)

Normes de qualité en relations publiques (dirigé par Bernard Poursuite du projet de recherche auprès des membres de l'Alliance des cabinets de relations publiques du Québec: analyse des résultats du sondage sur les normes de qualité.

Identification des variables culturelles dans l'utilisation des modèles et stratégies de communication (dirigé par André A. Lafrance)

Recherche visant à développer un instrument méthodologique qui permet d'identifier, à distance, les variables culturelles dont doivent tenir compte les intervenants canadiens dans un programme de formation aux communications organisationnelles se déroulant dans des pays de culture différente.

Vigie multirisques de Montréal (dirigé par Pierre Bérubé) Projet de développement d'un outil de communication des risques et d'information des intervenants en sécurité civile incluant des indicateurs inspirés des tableaux de bord de gestion. Projet développé sous l'égide du Centre d'études ODESIA sur les nouvelles technologies et les relations publiques, en collaboration avec le Centre de sécurité civile de Montréal.

Internet et crise (dirigé par Pierre Bérubé)

Élaboration de références et de lignes directrices pour l'utilisation d'Internet par les relationnistes dans un contexte de crise. Description de cas types et conseils pratiques.

Triangulation $d u$ discours public (dirigé par André A. Lafrance et Lise Chartier)

Projet de recherche mettant en lumière les convergences ou 
Chaire de relations publiques et communication marketing

divergences entre les messages tels que conçus par les communicateurs, tels que diffusés par les médias et tels que compris par les publics.

Inventaire de la recherche en communication municipale au Québec (dirigé par Bernard Dagenais)

Élaboration d'une revue de la littérature sur la communication municipale au Québec.

\section{Coordonnées}

Chaire en relations publiques, UQAM

Faculté de communication, local WB-3425

Case postale 8888, succursale Centre-ville

Montréal (Québec), H3C 3P8

Téléphone : (1)514-987-3000 poste 2613

Télécopieur : (1)514-987-0394

Courriel : crp@uqam.ca

Site Web : www.crp.uqam.ca 